\title{
The Impact of the Implementation of a New Selective Screening Program for Neonatal Hip Ultrasound in Cardiff and Vale University Health Board
}

\section{Poacher $\mathbf{A}^{1^{*}}$ and Carpenter $\mathrm{C}^{2}$}

${ }^{1}$ Welshbone, South Wales Orthopaedics Research Network, Wales, UK

${ }^{2}$ Paediatric Orthopaedic Surgery, University Hospital of Wales, Cardiff, United Kingdom

"Corresponding author: Mr Arwel Poacher, Welshbone, South Wales Orthopaedics Research Network, Wales, UK, CF14 4XN, Tel: 07503163584; E-mail: poachera@cardiff.ac.uk

Received date: January 25, 2018; Accepted date: February 06, 2018; Published date: February 08, 2018

Copyright: ( 2018 Poacher A, et al. This is an open-access article distributed under the terms of the Creative Commons Attribution License, which permits unrestricted use, distribution, and reproduction in any medium, provided the original author and source are credited.

\begin{abstract}
Objective: Developmental dysplasia of the hip (DDH) is a common and preventable cause of disability. Early detection of $\mathrm{DDH}$ ( $<3$ months) is associated with reduced risk of surgical treatment, hence in addition to the traditional clinical screening, NIPE guidelines advocate the use of selective screening of those with DDH associated risk factors. In 2016, Cardiff and Vale University Health Board (CAVUHB) implemented a selective screening program for $\mathrm{DDH}$. This research will determine the impact and up-to-date cost effectiveness of a selective screening for DDH.
\end{abstract}

Methods: A retrospective study of all patients born between 1st of January 2016 and the $31^{\text {st }}$ of December 2016, who underwent ultra-sonographic screening for DDH in CAVUHB. Ultrasounds were graded using Graf's classification, treatment outcomes were determined by patient's records and costings were based on 2016 NHS tariffs. The research took place at the University Hospital of Wales (UHW).

Results: Screening of those with risk factors for DDH, but a normal examination diagnosed $72 \%$ of all DDH cases and $38 \%$ of treated DDH cases. Screening for risk factors cost CAVUHB £98914, with a cost per favorable outcome, defined as early detection and successful treatment of DDH without surgical intervention, of £12364. The mean cost of DDH treatment of a patient missed by screening was $£ 14431$. All DDH cases were detected through breech presentation or family history risk factors. Screening only those with these risk factors is more cost effective and equally successful with a cost per favorable outcome of $£ 9095$.

Conclusion: The selective screening program was successful and cost effective in detecting and treating cases of $\mathrm{DDH}$. However, the screening process can be made more cost-effective without reducing DDH detection rate, by omitting statistically insignificant risk factors from the screening criteria. Early detection of DDH is important for effective treatment. CAVUHB cost-effectively reduced morbidity and potential surgical mortality within the neonatal population because of its selective screening program for $\mathrm{DDH}$.

Keywords: Orthopedic; Ultrasound; Dysplasia; Disability; Neonatal

\section{Introduction}

Developmental dysplasia of the hip (DDH) is recognised as a spectrum of abnormalities affecting the hip in infants and children. From the physiologically immature hip, to pathological acetabular dysplasia with or without dislocation or subluxation of the femoral head [1]. As a result, the prevalence of DDH varies from 1.6-28.5 cases per 1000 live births [2] due to the absence of a universal definition within the literature. DDH is a common and preventable cause of disability [3] and forms a large portion of paediatric orthopedic practice. A later diagnosis of DDH leads to an increased risk of surgical management and long-term complications such as early degenerative arthritis and gait disturbances $[4,5]$. These currently represent a large burden of care and DDH is reported as the underlying aetiology in approximately one third of hip replacements of those under 65 [6].

In January 2016, Cardiff and Vale University Health board (CAVUHB) implemented a selective screening ultrasound program for DDH, in accordance with Newborn and Infant Physical Examination
(NIPE) screening program guidelines [7]. Prior to 2016, CAVUHB used only the Ortolani and Barlow clinical examinations in primary care to screen for DDH. These tests are performed as part of the newborn and 6-week neonatal examinations with an abnormal examination resulting in referral for ultrasonographic assessment for $\mathrm{DDH}$.

The Ortolani and Barlow clinical examinations have very high specificity but poor sensitivity [8]. The Ortolani manoeuvre is around $60 \%$ sensitive and the Barlow manoeuvre has a positive predictive value (PPV) of only $22 \%$. The poor sensitivity of these tests is as a result of their inability to detect stable acetabular dysplasia which requires further ultrasound imaging for detection [9]. This high proportion of false negative results lead to a large proportion of DDH cases being missed. Therefore, without selective screening in CAVUHB, a substantial proportion of DDH cases were missed often requiring costly and invasive surgical treatments.

The importance of early detection and treatment has meant that nationwide clinical screening for DDH has been commonplace in the United Kingdom since the 1930's. National NIPE guidelines 
recommend ultrasound screening of neonates with risk factors for $\mathrm{DDH}$ and clinical screening at the newborn and 6-8 week neonatal check-ups. Common opinion describes that $60 \%$ of DDH is associated with factors including female sex, family history, breech presentation, caesarean section, foot deformities, intra uterine growth restriction (IUGR), and oligohydramnios.

These risk factors are derived from the Standing Medical Advisory Committee (SMAC) guidance [10], however, there are no references from the literature published around the time of release that can confirm this statement. Therefore, this guidance and our current understanding of the risk factors associated with DDH is most likely based on decades old expert opinion/level 5 evidence. These risk factors are included in the CAVUHBs screening criteria to identify those at risk. However, if any of these risk factors are insignificant then resources are wasted and an increased rate of overtreatment may be seen. Hence, we have conducted analysis of risk factor based referrals and their association with outcome and treatment. Furthermore, as there is no up-to-date data on the cost effectiveness or impact of the inclusion of individual risk factors in the screening criteria for a large UK population, we conducted a cost benefit analysis of this new screening program.

\section{Methodology}

A retrospective study of all neonatal hip ultrasound scans within CAVUHB between $1^{\text {st }}$ January 2016 and $31^{\text {st }}$ December 2016. A reporting radiologist, an orthopedic consultant, and a medical undergraduate each separately classified the scans according to Graf [11]. Demographics such as age and sex were recorded alongside alpha angle, Graf classification and treatment success. Successful treatment was defined as the hip being measured as a Graf type I hip ( $>60$ degrees) on an ultrasound, or a reported acetabular angle of $\leq 30$ degrees on X-ray, as defined by Tönnis [12].

Treatment information of each patient was obtained from their hospital notes. Forty-four patients were excluded due to lack of information regarding their referral. A further three patients were excluded due to a lack of follow up or having not completed treatment. Costings for clinic appointments, ultrasounds, and treatments were taken from the NHS 2016/17 National and National tariff workbook or if unavailable the most recent appropriate literature [13].

\section{Results}

Between the $1^{\text {st }}$ of January 2016 and the $31^{\text {st }}$ of December 2016, there were 5,288 live births within CAVUHB. Five hundred ninety-two (11\%) were referred for clinical and radiographic assessment of the hip, $106(2 \%)$ had a diagnosis of DDH, and $21(0.39 \%)$ received treatment for developmental dysplasia of the hip of the 21 patients that required treatment, $10(47.6 \%)$ presented $<6$ weeks of age, $8(38.1 \%)$ were aged between 6-24 weeks and the remaining 3 patients (14.3\%) presented for the first time $>24$ weeks of age. The low numbers of late-presenting patients requiring treatment highlights the effectiveness of the selective screening program in detecting treatable $\mathrm{DDH}$.

Treatment increased in complexity with the age of diagnosis. Of those presenting at $<6$ weeks, $42.9 \%(\mathrm{n}=3)$ required surgical reduction whilst of those that presented over 24 weeks, $100 \%(n=3)$ required surgical reduction (Figure 1). Therefore, if the DDH is detected early, ideally $<6$ weeks, there is a higher likelihood that conservative methods will be sufficient to treat DDH thus reducing the cost and associated morbidity associated with surgical treatment.
The cost of treatment increased with the age of presentation. The group presenting $<6$ weeks the average cost of treatment was $£ 4354$ (range $£ 1516$ - $£ 14454$ ) and for those presenting over $>24$ weeks the average cost of treatment was $£ 14431$ (range $£ 10724$ - $£ 20876$ ).

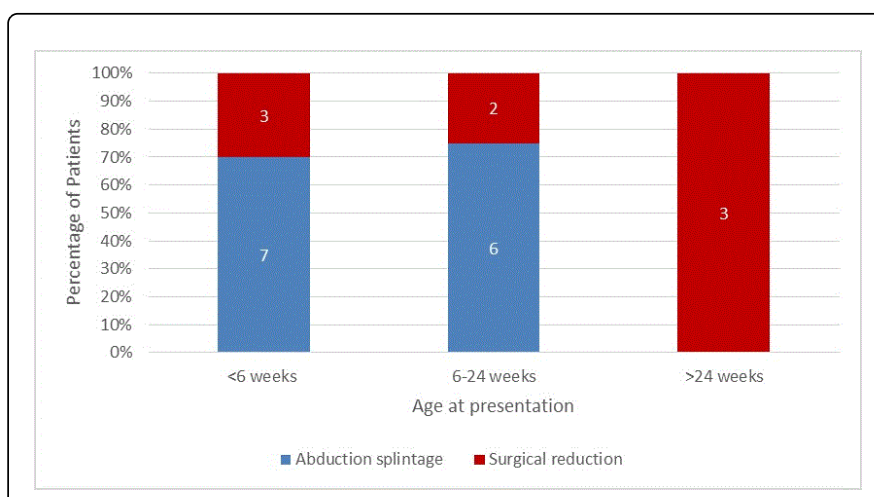

Figure 1: A bar chart representation of the proportion of treatments, either abduction splintage or surgical reduction, undertaken within each age at presentation category. The number within each designated region represents the number of patients.

Five-hundred forty-eight patients were included in this study, of these $416(75.9 \%)$ were referred due to the presence of risk factors. $78.1 \%(n=350)$ of the bilateral Graf type I hips, $77.2 \%(n=79)$ of type IIa-IV hips and $38.1 \%(n=8)$ of those that required treatment were referred due to the presence of risk factors (Figure 2).

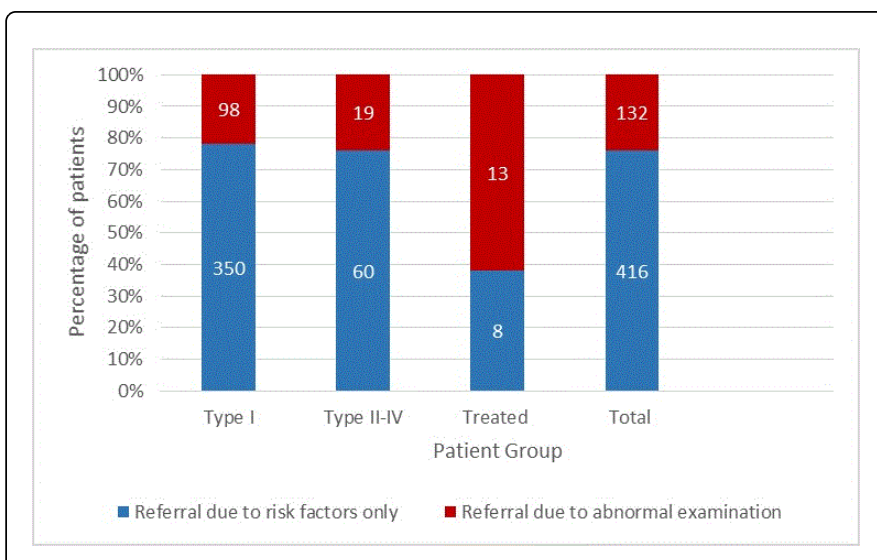

Figure 2: A bar chart representation of the proportion of referrals made due to abnormal examination or the presence of risk factors. The number within each designated region represents the number of patients referred within the set criteria.

As the severity of DDH increased, the reason for referral was more likely to be abnormal examination. In those with grade II hips the rate of referral due to risk factor was $79.4 \%(\mathrm{n}=77)$ but in grade IV hips it was $20 \%(n=1)$ (Figure 3). 
Citation: Poacher A, Carpenter C (2018) The Impact of the Implementation of a New Selective Screening Program for Neonatal Hip Ultrasound in Cardiff and Vale University Health Board. J Arthritis 7: 265. doi:10.4172/2167-7921.1000265

Page 3 of 5

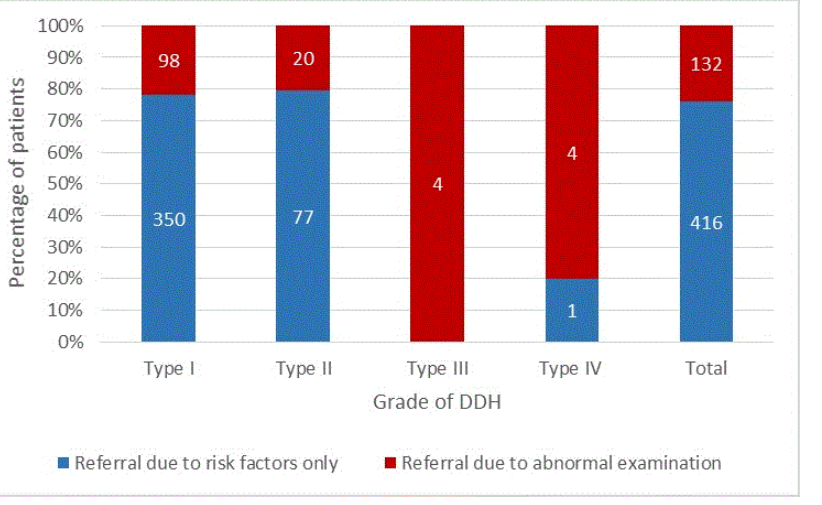

Figure 3: A bar chart representation of the proportion of referrals made due to abnormal examination or the presence of risk factors by Grade of $\mathrm{DDH}$ at presentation. The number within each designated region represents the number of patients referred within the set criteria.

Inclusion of risk factors into screening criteria increased the number of ultrasounds performed at UHW significantly. From a mean of 189 (range 171-214) scans between 2010-2015, to 721 scans in 2016 (Figure 4). This four-fold increase in the number of scans and clinic appointments increased the resources dedicated to DDH screening and diagnosis.

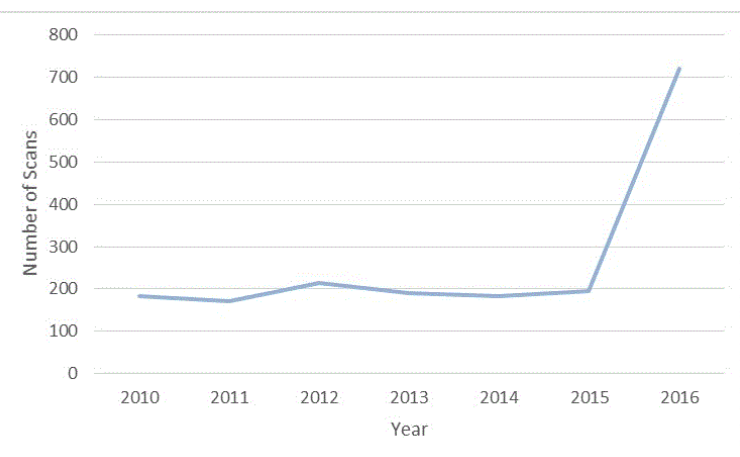

Figure 4: The number of ultrasound scans performed in CAVUHB for DDH screening per year (2010-2016), highlighting the significant increase in 2016 when a selective screening program was implemented.

The estimated total cost of the screening program, including clinic appointments and treatment was $£ 245,068$ (Table 1 ).

\begin{tabular}{|l|l|l|l|}
\hline Patient contact & Number $(\mathbf{n})$ & Cost $(\boldsymbol{£})$ & Total cost (£) \\
\hline First clinic appointments & 592 & 145 & 86130 \\
\hline Follow up clinic & & & \\
\hline appointments & 129 & 94 & 13818 \\
\hline Ultrasound & 721 & 43 & 31003 \\
\hline Total cost of screening & & & 130951 \\
\hline
\end{tabular}

\begin{tabular}{|c|c|c|c|}
\hline \multicolumn{4}{|l|}{ treatment } \\
\hline Pavlik Harness & 15 & 1515.88 & 22738.2 \\
\hline closed reduction & 6 & 3730 & 22380 \\
\hline open reduction & 4 & 6731 & 26924 \\
\hline Pelvic osteotomy & 1 & 6138 & 6138 \\
\hline EUA & 9 & 3993 & 35937 \\
\hline Total cost of treatment & & & 114107.2 \\
\hline $\begin{array}{l}\text { Total cost of screening and } \\
\text { treatment }\end{array}$ & & & 245068.2 \\
\hline
\end{tabular}

Table 1: A table representing the cost breakdown by appointment type and number with treatment of all patients included in the DDH screening program from 2016.

The total cost of screening and treatment for those with risk factors was $£ 109,041,44.5 \%$ of the total cost of the screening and treatment of DDH in CAVUHB during 2016. The total cost of screening for DDH in CAVUHB in 2016 was $£ 130951$. Of this, the cost screening for risk factors was $£ 96914$ (Table 2), therefore screening for risk factors made up $74 \%$ of the total cost of screening.

\begin{tabular}{|c|c|c|c|}
\hline \multicolumn{2}{|l|}{ All Risk Factors } & \multirow[b]{2}{*}{ Cost (£) } & \multirow[b]{2}{*}{ Total (£) } \\
\hline Patient contact & Number (n) & & \\
\hline Ultrasounds & 541 & 43 & 23263 \\
\hline First clinic appointment & 447 & 145 & 64815 \\
\hline Follow up appointment & 94 & 94 & 8836 \\
\hline Total cost of screening & & & 96915 \\
\hline \multicolumn{4}{|l|}{ Treatment } \\
\hline Pavlik Harness & 8 & 1515.88 & 12127.04 \\
\hline Total cost of treatment & & & 12127.04 \\
\hline $\begin{array}{l}\text { Total cost of screening and } \\
\text { treatment }\end{array}$ & & & 109041 \\
\hline
\end{tabular}

Table 2: A table showing the cost breakdown of screening for all risk factors by appointment, type and number, and treatment of all patients who were referred due to risk factors in the $\mathrm{DDH}$ screening program 2016.

Eight patients required treatment following referral due to their risk factors, with the total cost of screening being $£ 96,914$ equating to a cost per favorable outcome (defined as early detection and successful treatment without surgical intervention) of $£ 12,114$. Breech and family history were the most common reasons for referral across all patient groups except for bilateral type 1 (normal) hips. Had only breech and family history been included in the referral criteria, all of the treated cases of DDH screened due to risk factors would have still been detected. Whilst, incurring a reduced total cost of screening of $£ 72758$ (Table 3), and a cost per favorable outcome of $£ 9,095$. Therefore, including only risk factors that are part of the referral criteria reduced the cost of screening by $£ 24,156$ (25\%), in 2016. As such, CAVUHB’s selective screening program was significantly more cost-effective 
without increasing the risk of late-presenting DDH in the neonatal population.

\begin{tabular}{|l|l|l|l|}
\hline \multicolumn{4}{|l|}{ Breech and Family History Screening only } \\
\hline Patient contact & Number (n) & Cost (£) & Total (£) \\
\hline Ultrasounds & 406 & 43 & 17458 \\
\hline First clinic appointment & 336 & 145 & 48720 \\
\hline Follow up appointment & 70 & 94 & 6580 \\
\hline Total cost of screening (£) & & & 72758 \\
\hline Treatment & & & \\
\hline Pavlik Harness & 8 & 1515.88 & 12127.04 \\
\hline $\begin{array}{l}\text { Total cost of screening and } \\
\text { treatment (£) }\end{array}$ & & & 84885.04 \\
\hline
\end{tabular}

Table 3: A table outlining the cost breakdown by appointment, and treatment of all patients who were referred due to breech or Family history risk factors in the DDH screening program for 2016.

\section{Discussion}

The earlier a child presents the less likely they are to require costly and invasive treatments [14] thus highlighting a need for early identification and treatment of DDH. All late presenting patients in this study had grade IV DDH that required open reduction, whilst the majority of those who were referred early ( $<6$ weeks), were treated with simple abduction splintage techniques such as the Pavlik harness. The patients who presented late did not have the risk factors required for selective screening and their DDH was detected much later, requiring more costly and invasive treatment. The only solution to these missed cases is the implementation of a universal screening program. The debate between universal and selective screening programs for DDH is ongoing [15] but current NIPE standards recommend a selective screening program only.

The establishment of new selective screening programmes incurs costs which can be rationalised by the inclusion of the most financially cost effective and statistically relevant risk factors. Within our cohort of those screened due to positive risk factors and positive for a diagnosis of $\mathrm{DDH}$, all were referred either because of a third trimester breech presentation or due to a $1^{\text {st }}$ degree family history. Had screening criteria only included referral due to breech presentation or $1^{\text {st }}$ degree family history the cost per favorable outcome would be $£ 9095$, 39\% cheaper than the average cost of treating a missed case of DDH and $25 \%$ cheaper than the current screening programs. Risk factors such as oligohydramnios, multiple gestations, and foot deformities (Congenital Talipes Equinovarus) were included in the referral criteria due to previous experience within the unit, however, may not be statistically significant risk factors for DDH [16]. Therefore, removal of these risk factors from the screening criteria may increase cost-efficiency and allows for more effective reallocation of much needed specialist time and resources. The selective screening for DDH has not been universally cost effective [17] and we have shown that by removing less significant risk factors that do not have a robust evidence base to justify their use, selective screening can be more cost-effective (25\%) without increasing risk of late presenting cases of DDH.
The cost of screening of all newborns with risk factors per favorable outcome was $£ 12114$ and the cost of surgical intervention was $£ 14431$. However, this is based purely on the tariff for procedures and excludes any additional costs such as complications, clinic follow-up, or excess hospital stay, and is therefore a substantial underestimate. Even whilst underestimating the cost of surgical treatment, the cost of screening for risk factors per favorable outcome is $18 \%$ lower than the estimated cost of treating a missed case of DDH. As such, screening is not only cost effective, but avoids the physical and social consequences of invasive surgery.

A low rate of successful detection of DDH was demonstrated by neonatal clinical examination. Only $22.8 \%$ of patients with ultrasound confirmed DDH were referred with an abnormal examination, supporting the contention that both the newborn and six-week clinical screening checks are unreliable. However, ultrasonographic diagnosis of DDH has a higher prevalence of abnormality than clinical diagnosis, raising the possibility of an over diagnosis of the condition which may have led to over treatment. The Medical Research Council (MRC) working party on congenital dislocation of the hip estimated the incidence of surgical procedures for treatment of DDH as 1-2 per thousand live births in a clinically screened population [10]. In this cohort, 1.5 per thousand live births required surgical treatment for $\mathrm{DDH}$, suggesting that there is no overtreatment of DDH because of the selective screening program. This contradicts a review of Shipman et al. [18] which claims that selective screening for DDH may lead to over-treatment. Currently training for the neonatal hip examination consists of two and a half hours of theoretical training and practice experience with a model. Therefore, one area for reducing the proportion of missed cases alongside selective or universal screening would be to increase the training and experience of clinicians in a controlled environment.

One limitation of this study is that the age at presentation was calculated from the date of birth not expected delivery date due to an absence of available data. In addition, as this data was collected from only CAVUHB we could not account for geographical variation of $\mathrm{DDH}$ incidence. However, CAVUHB contains a large, diverse population that can reasonably be applied to other UK and European populations.

In conclusion, the age of presentation to medical services increases alongside the complexity and invasiveness of treatment. Thus, late presentation causes increased morbidity to patients and incurs a vastly higher cost of treatment. This, in conjunction with the cost effectiveness of CAVUHB's selective screening program clarifies the success of its implementation. However, the screening process can be made more cost-effective without reducing rates DDH detection, by omitting statistically insignificant risk factors from the screening criteria.

\section{References}

1. Mahan ST, Katz JN, Kim YJ (2009) To screen or not to screen? A decision analysis of the utility of screening for developmental dysplasia of the hip. J Bone Joint Surg Am 91: 1705-1719.

2. Dezateux C, Rosendahl K (2007) Developmental dysplasia of the hip. Lancet 369: 1541- 1552.

3. Gulati V, Eseonu K, Sayani J, Ismail N, Uzoigwe C, et al. (2013). Developmental dysplasia of the hip in the newborn: A systematic review. World J Orthop 18: 32-41. 
Citation: Poacher A, Carpenter C (2018) The Impact of the Implementation of a New Selective Screening Program for Neonatal Hip Ultrasound in Cardiff and Vale University Health Board. J Arthritis 7: 265. doi:10.4172/2167-7921.1000265

Page 5 of 5

4. Dunn PM, Evans RE, Thearle MJ, Griffiths HED, Witherow PJ (1985) Congenital dislocation of the hip: early and late diagnosis and management compared. Arch Dis Child 60: 407-414.

5. Bache CE, Clegg J, Herron M (2002) Risk factors for developmental dysplasia of the hip: ultrasonographic findings in the neonatal period. J Pediatr Orthop B 11: 212-218.

6. Furnes O, Lie SA, Espehaug B, Vollset SE, Engesaeter LB, et al. (2001) Hip disease and the prognosis of total hip replacements. A review of 53,698 primary total hip replacements reported to the Norwegian Arthroplasty Register 1987-99. J Bone Joint Surg Br 83: 579-586.

7. Public Health England (2016) Newborn and Infant Physical Examination (NIPE) screening program standards.

8. Paton RW, Hinduja K, Thomas CD (2005) The Significance of at-risk Factors in Ultrasound Surveillance of Developmental Dysplasia of the Hip: A Ten Year Prospective Study. The J Bone Joint Surg Br 87: 1264-1266.

9. Murray KA, Crim JR (2001) Radiographic Imaging for Treatment and Follow-up of Developmental Dysplasia of the Hip. Semin Ultrasound CT MR 22: 306-340.

10. The Standing Medical Advisory Committee (SMAC) (1996) Screening for the detection of congenital dislocation of the hip. Department of Health and Social Security, London.
11. Graf R (1983) New possibilities for the diagnosis of congenital hip joint dislocation by ultrasonography. J Pediatr Orthop 3: 354-359.

12. Tönnis D (1976) Normal Values of the Hip Joint for the Evaluation of Xrays in Children and Adults. Clin Orthop Relat Res 119: 39-47.

13. Price KR, Dove R, Hunter JB (2013) Current screening recommendations for developmental dysplasia of the hip may lead to an increase in open reduction. Bone Joint J 95: 846-850.

14. Pollet V, Percy V, Prior H (2017) Relative Risk and Incidence for Developmental Dysplasia of the Hip. J Pediatr 181: 202-207.

15. Shorter D, Hong T, Osborn D (2013) Cochrane Review: Screening programmes for developmental dysplasia of the hip in newborn infants. Evid Based Child Health 8: 11-54.

16. Hundt M, Vlemmix F, Bais J, Hutton E, Groot C, et al. (2012) Risk factors for developmental dysplasia of the hip: a meta-analysis. Eur J Obstet Gynecol Reprod Biol 165: 8-17.

17. Sakthivel VK, Goddard M, Sabouni MY, Clarke NMP (2006) Selective Screening for DDH in Wessex-the Southampton experience. Orthopaedic Proceedings 88: 435.

18. Shipman SA, Helfand M, Meyer VA, Yawn BP (2006) Screening for developmental dysplasia of the hip: a systemic literature review for the US preventative services task force. Pediatrics 117: e557-576 